\title{
Progress in neutrino astronomy
}

\author{
Carsten $\operatorname{Rott}^{1}{ }^{1}$
}

Received: 6 October 2020 / Accepted: 29 October 2020 / Published online: 19 March 2021

(c) The Author(s) 2021

\begin{abstract}
The dream of observing our universe through neutrinos is rapidly becoming a reality. More than three decades after the first observation of neutrinos from beyond our solar system associated with Supernova SN1987A, neutrino astronomy is in the midst of a revolution. Extraterrestrial neutrinos are now routinely detected, following the discovery of a high-energy diffuse astrophysical neutrino flux in 2013. The detection of a high-energy neutrino in coincidence with a flaring blazar in 2017 has brought the field rapidly into the multi-messenger science era. The latest developments in the field of neutrino astronomy are reviewed and prospects with current and future detectors discussed. Particular emphasis is put on domestic programs in neutrino astronomy and the possibility to construct a large neutrino observatory in Korea.
\end{abstract}

Keywords Neutrino astronomy $\cdot$ IceCube $\cdot$ KNO

\section{Introduction}

Through astronomical observations cover an ever broader range of the electromagnetic wavelength spectrum, we have gained remarkable insights into the workings of the universe. More recently, astroparticle physics has allowed us to expand the spectrum of the astronomical messenger particles to acquire an even deeper understanding of the cosmos. Despite tremendous progress, the most extreme environments in the cosmos, such as those near supermassive black holes or exploding stars, remain shrouded in mystery.

Energetic radiation in the form of gamma-rays is of special interest to explore these extreme phenomena. However, as the universe is filled with infra-red photons, $\gamma^{\mathrm{IR}}$, and cosmic microwave background radiation, $\gamma^{\mathrm{CMB}}$, that interact resonantly with high-energy gamma-rays. Thus, the observable distance that is accessible with energetic radiation is limited. High-energy gamma-rays are attenuated due to resonant interactions with the cosmic microwave background (CMB) radiation $\left(\gamma+\gamma^{\mathrm{CMB}} \rightarrow e^{+} e^{-}\right)$and interactions with infra-red stellar background light producing $\gamma+\gamma^{\mathrm{IR}} \rightarrow e^{+} e^{-}$. This interaction with the extragalactic background light (EBL) and CMB photons results in an attenuation of the

Carsten Rott

rott@skku.edu

1 Department of Physics, Sungkyunkwan University, Suwon 16419, Korea gamma-ray flux from distant sources [1] and restricts the horizon observable with gamma-rays, as shown in Fig. 1. Cosmic rays, deflected by magnetic fields and interacting with the cosmic microwave background, resulting in the Greisen-Zatsepin-Kuzmin (GZK) cut-off, are similarly limited.

The only messenger particle capable of directly exploring the energetic universe and shedding light on the underlying particle processes is the neutrino. For this reason, highenergy neutrinos are essential to determine the origin of the high-energy cosmic rays and explore the most extreme environments in our universe. The study of high-energy neutrinos is a topic with rich connections across particle physics and astrophysics. It also plays an essential role in answering some of the most fundamental questions of our time, such as phenomena beyond the Standard Model of particle physics, the nature of dark matter, and determining the properties of neutrinos. The impact and discovery potential in this field remains extremely high, and following the observation of high-energy astrophysical neutrinos, new opportunities have emerged to study rare phenomena through the propagation of neutrinos from distant sources.

This article is structured in the following way: in Sect. 2, current and next generation neutrino telescopes are reviewed. The quest to detect neutrinos of astrophysical with these neutrino telescopes is discussed in Sect. 3, and science with large-volume neutrino detectors in Sect. 4, before concluding in Sect. 5. 
Fig. 1 The length scale of the observable Universe and the energy scale $\mathrm{E}$ of neutrinos, together with potential and detected astrophysical sources, are shown. The gamma-ray horizon indicates the distance at which gamma-rays are severely attenuated due to the interaction with photons from the extragalactic background light or $\mathrm{CMB}$

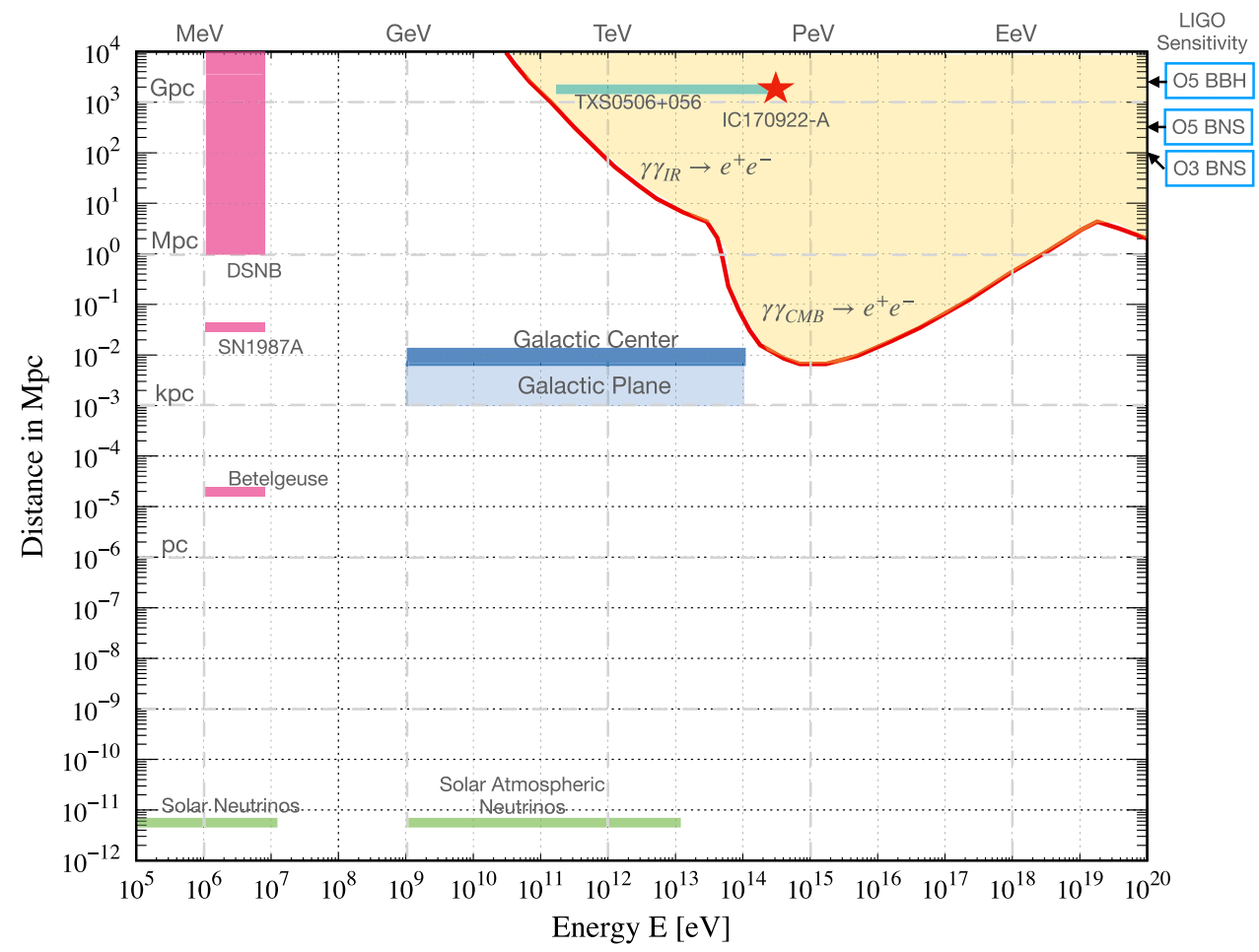

\section{Neutrino telescopes}

The field of astroparticle physics is in the midst of a revolution following the discovery of high-energy astrophysical neutrinos [2, 3] and the recent observation of neutrino emissions in the direction of blazar TXS 0506+056 [4, 5] by the IceCube Neutrino Observatory. These scientific breakthroughs, combined with the very diverse and high impact science programs at neutrino telescopes, have triggered a broad interest in neutrino telescope science. International efforts are underway to construct new telescopes in Europe (KM3NeT-ORCA near Marseille and KM3NeT-ARCA near Sicily) [6], in Siberia (BaikalGVD) [7], and in Antarctica (IceCube-Gen2) [8]; a test site is being explored at the Ocean Networks Canada in the Pacific Ocean (P-ONE) [9]. Domestically plans for a Korean Neutrino Observatory (KNO) are under intense discussion.

\subsection{IceCube neutrino telescope}

The IceCube Neutrino Observatory is the world's largest neutrino detector. Since completion in 2010, it has executed a highly successful scientific program over a broad science scope ranging from the groundbreaking discovery of high-energy astrophysical neutrinos, to a breakthrough candidate source detection in association with a blazar flare, to measurements of neutrino particle properties and world-leading bounds on dark matter properties. IceCube can detect neutrinos from $10 \mathrm{GeV}$ to beyond $\mathrm{PeV}$ energies, as well as Galactic supernova burst neutrinos at $10 \mathrm{MeV}$. The funded IceCube Upgrade (2022/2023), and prospects for creating a IceCube-Gen2 facility, will expand the energy coverage and the scientific scope even further.

The IceCube Neutrino Telescope (see Fig. 2) instruments a cubic kilometer of ultra-pure Antarctic ice at the

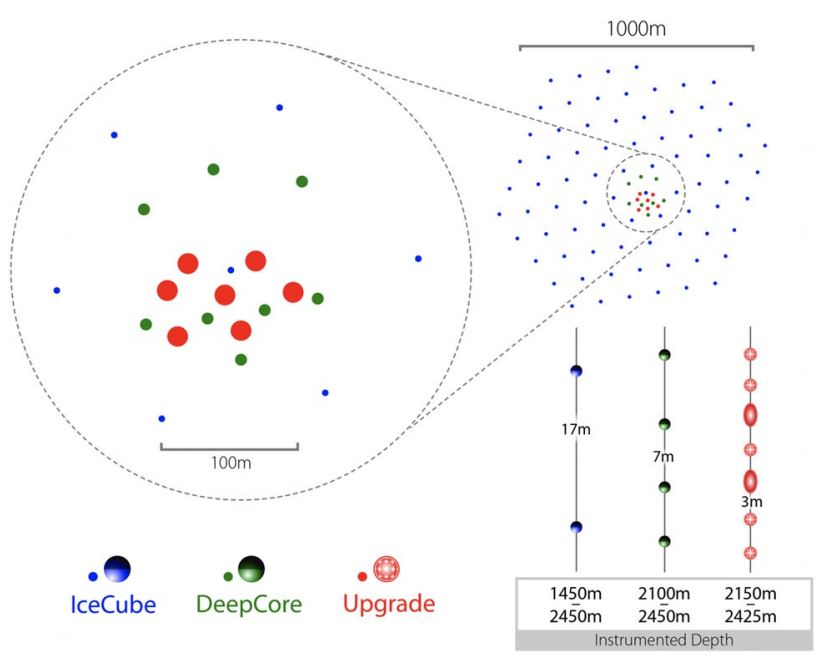

Fig. 2 Geometry of the IceCube neutrino telescope (blue), the IceCube Upgrade string array (red), and the IceCube DeepCore subarray strings (green) 
geographic South Pole with more than 5000 optical sensor modules. The optical modules detect neutrinos through Cherenkov light emission of secondary particles produced in neutrino interactions. Competitive measurements of neutrino oscillation parameters, leading dark matter searches, cosmic-ray physics, and atmospheric and astrophysical neutrino measurements are made possible by IceCube's large dynamic range and energy coverage.

\subsubsection{IceCube Upgrade}

The IceCube Upgrade, consisting of an additional seven detector strings at the bottom center of IceCube, will be deployed in $2022 / 2023$. These strings will dramatically boost IceCube's performance at the $10 \mathrm{GeV}$ energy scale, enabling fundamental physics such as the world's most stringent test of unitarity in the tau sector of the PMNS matrix, as well as dark matter searches at mass ranges of a few $\mathrm{GeV}$ that are not well constrained. The Upgrade provides an opportunity to re-calibrate the IceCube detector with new calibration devices [10]. Improved optical ice models obtained in this process could be retroactively applied to the already collected IceCube data. The IceCube Upgrade shares many scientific objectives with the PINGU concept [11], but requires fewer sensor modules due to compromises on the low-energy threshold.

Each IceCube Upgrade string will be equipped with approximately 100 optical modules, consisting of a roughly equal number of multi-PMT digital optical modules (mDOMs) [12] and dual optical sensors in an Ellipsoid Glass for Gen2 (D-EGGs) [8]. For the Upgrade, the

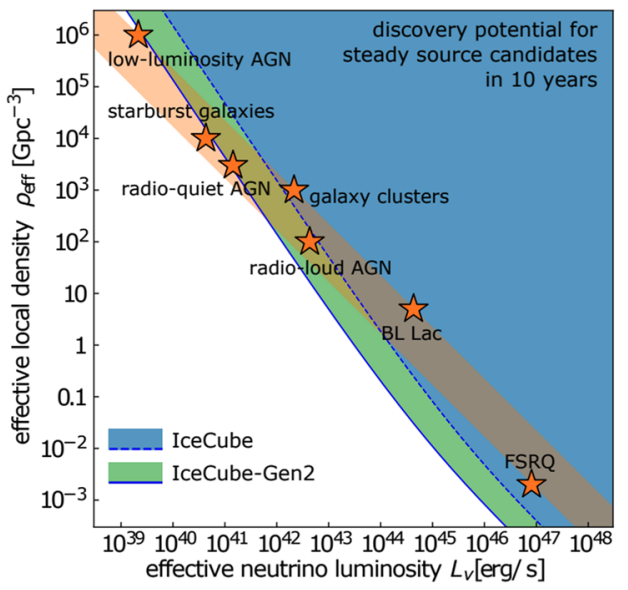

Fig. 3 Left: comparison of the diffuse neutrino emission (solid orange band) to the effective local density $\rho_{\text {eff }}$ and neutrino luminosity $L_{v}$ of various extragalactic neutrino source populations. Several candidate source populations are indicated by the required neutrino luminosity to account for the full diffuse flux [13, 14]. The width of the band is given by the redshift evolution of the sources. The lower (upper) edge of the band assumes rapid (no) redshift evolution. The optical modules will primarily be deployed at depth between $2150 \mathrm{~m}$ and $2425 \mathrm{~m}$ below the surface of the South Pole ice, where the glacial ice is clearest. At these depths, the greatly reduced atmospheric muon background can be vetoed efficiently by the surrounding detector array.

\subsubsection{IceCube-Gen2}

IceCube-Gen2 will be a wide-band neutrino observatory that combines optical and radio technologies for the detection of neutrinos from $\mathrm{MeV}$ to $\mathrm{EeV}$ energies [8]. A surface array will be utilized for cosmic-ray physics and for vetoing atmospheric neutrino events. A significantly larger in-ice optical sensor array will enhance the neutrino event rate in the upper $\mathrm{TeV}$ energy range by an order of magnitude over the current detector. The construction of a low-energy core is already progressing with the IceCube Upgrade. The sensitivity to transient and steady astrophysical neutrino sources is shown in Fig. 3.

\subsection{Neutrino telescopes in the Mediterranean}

Following more than 12 years (since 2008) of successful operation of the ANTARES neutrino telescope [17] in the Mediterranean sea, the construction of the KM3NeT detector [6] is now underway. The detector is deployed at two sites. KM3NeT ARCA (Astroparticle Research with Cosmics in the Abyss) is being constructed near Capo Passero in Sicily at a depth of $3.5 \mathrm{~km}$ and a distance of $100 \mathrm{~km}$ from shore. KM3NeT ORCA (Oscillation Research with Cosmics in the Abyss) is being constructed close to the ANTARES

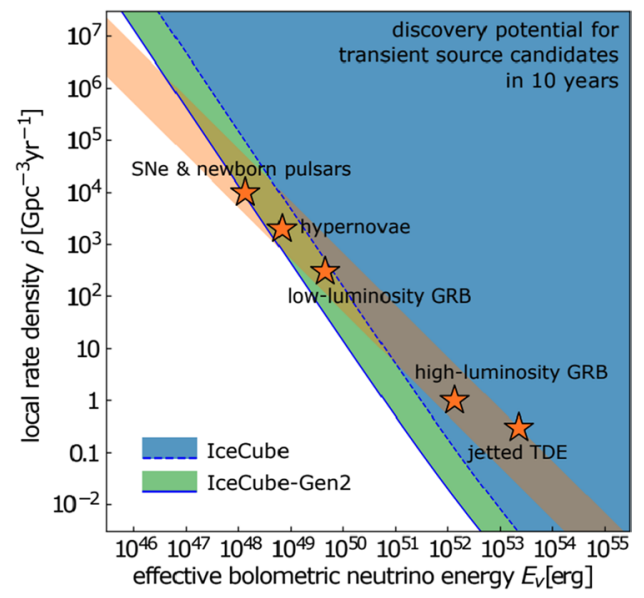

blue and the green regions indicate the ability of IceCube and IceCube-Gen 2 to discover one or more sources of the respective populations $\left(E^{2} \phi_{\nu_{\mu}+\bar{\nu}_{\mu}} \simeq 10^{-12} \mathrm{TeV} / \mathrm{cm}^{2} / \mathrm{s}\right.$ in the Northern Hemisphere [15]). Right: comparison for transient neutrino sources defined by their local rate density and effective bolometric neutrino energy [16]. Figures are from Ref. [8] 
site at a depth of $2.5 \mathrm{~km}$ and at a distance of about $40 \mathrm{~km}$ from Toulon, France.

The first ARCA string was deployed in December 2015, and two strings were operational till November 2019. Construction will restart in Fall 2020 with the goal to complete the construction of two 115 string clusters by 2026. The ORCA string deployment started in September 2017 and has had six strings operational since January 2020 . The goal is to complete the deployment of a total of 115 strings in 2024 [18].

\subsection{Lake Baikal Gigaton volume neutrino telescope}

The deep underwater neutrino telescope Baikal Gigaton Volume Detector (Baikal-GVD) is expected to achieve an instrumented volume of about two cubic kilometers using an array of $\sim 10.000$ optical modules. The first phase, GVD-1, with an effective volume of $0.4 \mathrm{~km}^{3}$, is planned for completion in 2020-2021. It will comprise 8 clusters with 288 optical modules each [7]. Sensors are deployed at a depth of 750-1275 m. The Baikal optical sensor modules consist of a pressure-resistant glass sphere equipped with a 10 -inch Hamamatsu R7081-100 PMT with a quantum efficiency of $\sim 35 \%$ [7].

\section{Search for high-energy extraterrestrial neutrinos}

\subsection{Diffuse astrophysical neutrino flux}

In 2013, IceCube published the first measurement of an isotropic diffuse astrophysical neutrino flux [2, 3]. The initial analysis used for this discovery focused on a sample of neutrino events detected in the inner detector volume with low background contamination. Since the discovery, several independent analyses have confirmed the diffuse flux, and a relatively consistent picture has emerged, with different event samples covering a variety of energy ranges, event topologies, and sky hemispheres. While the different analyses favor slightly different spectral indices and normalizations, the differences are not statistical significant. The mild tension between different analyses could be an indication that a simple power-law fit is not sufficient to describe the astrophysical neutrino flux spectrum. Spectral features, such as a break in the power-law spectrum or a cut-off, will be resolved with higher statistics samples. With the present available statistics, additional spectral features do not significantly improve fit results [19]. The inconsistency with a power-law spectrum is also a guarantee for measurements with future neutrino telescopes to shed new light on the astrophysical neutrino flux or to discover unexpected physics beyond the Standard Model. Figure 4 shows the latest fit of

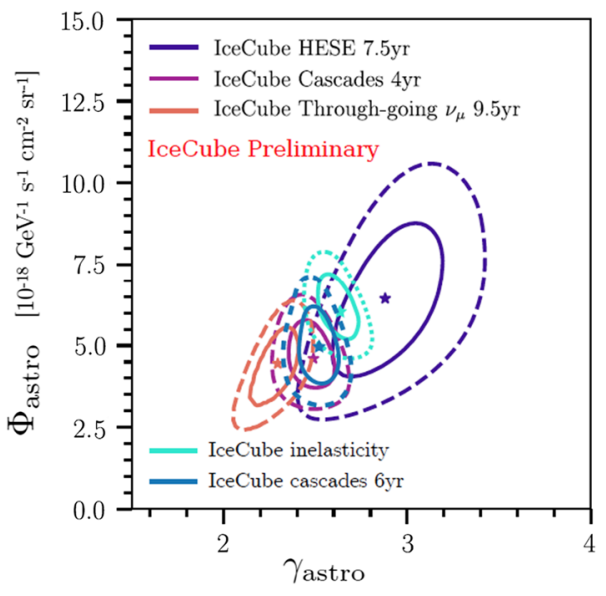

Fig. 4 Fit of the diffuse astrophysical neutrino flux using a power law with the spectral index $\gamma^{\text {astro }}$. Figure is taken from Ref. [20]

the IceCube flux modeled with a simple power-law spectrum with a spectral index $\gamma^{\text {astro }}$ and flux normalization $\phi_{\text {astro }}^{100 \mathrm{TeV}}$ [20]. The flux is normalized at $100 \mathrm{TeV}$ and described by $\phi\left(E_{v}\right)=\phi_{\text {astro }}^{100 \mathrm{TeV}}\left(\frac{E_{v}}{100 \mathrm{TeV}}\right)^{-\gamma_{\text {astro }}}$. Different analyses include 6-year cascade [21], muon neutrinos [22], measurements using the inelasticity distribution of multi-TeV neutrino interactions [23], and the High-Energy Starting Events (HESE) sample [24].

\subsection{Extraterrestrial neutrino sources}

\subsubsection{Search for counterparts of high-energy neutrino events}

On September 22, 2017, a neutrino alert that spurred multimessenger follow-ups from radio to $\gamma$-rays was issued by IceCube (IC-170922A). Fermi-LAT and MAGIC identify a spatially coincident flaring blazar (TXS 0506+056). The chance probability of a Fermi-IceCube coincident observation was determined to be $3 \sigma$ based on the historical IceCube sample and known Fermi-LAT blazars. The redshift of the candidate source TXS $0506+056$ was determined to be $z=0.3365$ [25].

Following the coincident detection of a high-energy neutrino with the flaring blazar, 9.5 years of archival data in the direction of TXS 0506+056 were evaluated, and an excess of $13 \pm 5$ events above background was observed during the period from September 2014 to March 2016 [5]. This observation is inconsistent with the background-only hypothesis at the $3.5 \sigma$ and is independent of the $3 \sigma$ associated with the IceCube-170922A alert. Note that during the neutrino flare in 2014-2016, TXS 0506+056 was not active in gammarays. This might not be surprising, as an efficient neutrino source is opaque to gamma-rays, since neutrino production 
requires a large target density with a high opacity to protons. The time-integrated neutrino spectrum is approximately $E^{-2.1}[5]$.

The association of a radio-emitting tidal disruption event with a high-energy neutrino (IC191001 A-detected on Oct 1, 2019) was recently reported as part of a systematic search for optical counterparts to high-energy neutrinos at the Zwicky Transient Facility (ZTF) [26]. The probability of a random coincidence was determined to be $0.5 \%$, or about 2.6 $\sigma$. Tidal Disruption Events (TDEs) are rare transients that occur when stars pass by close to a supermassive black hole (SMBH) and get pulled apart by tidal forces. Powerful jets and outflows generated in TDEs have been suggested as possible sites producing high-energy neutrinos [27-29].

The association between IC-170922A and the blazar TXS 0506+056 highlights the importance of real-time observations for identifying astrophysical neutrino sources. IceCube is well suited to generate alerts for other observatories or to follow up rapidly those generated by other instruments. IceCube has a near- $100 \%$ duty cycle, a $4 \pi$ steradian field of view, and an excellent sensitivity over many decades of energy.

\subsubsection{Search for steady point sources}

An unbinned likelhood analysis is used to search for clustering of muon-neutrino events from steady (constant flux) point sources [15]. The analysis relies on IceCube's excellent angular reconstruction capabilities that achieve a median angular resolution of muon-neutrino events of $0.3^{\circ}\left(1^{\circ}\right)$ at $1 \mathrm{PeV}(1 \mathrm{TeV})$. A full sky scan with nearly 500,000 neutrinos events collected between 2009 and 2017 found no evidence for neutrino sources or any significant excess in an a priori candidate source candidate list consisting of pulsar wind nebulae (PWNe), star formation regions (SFRs), supernova remnants (SNRs), starburst/radio galaxies (SRGs), X-ray binaries (XRBs) and micro-quasars (MQs), unidentified gamma-ray sources, and active galactic nucleus (AGN) type objects such as BL Lacertae (BL Lacs) and flatspectrum radio quasars (FSRQs) [15]. IceCube's most recent time-integrated search for neutrino sources covered 10 years of data collected from 2008 to 2018 [30]. The most significant hotspot was found at $0.35^{\circ}$ from NGC 1068 (Active galaxy of type Seyfert II), which was also one of 110 predefined candidate sources consisting of Galactic and extragalactic objects. Based on the Northern sky source catalog, the neutrino excess near NGC 1068 is inconsistent with a background-only hypothesis at $2.9 \sigma$ [30]. The neutrino flux from the sources in the Northern source catalog is inconsistent with the background-only hypothesis at $3.3 \sigma$. The present excess can primarily be traced back to four candidate sources: NGC 1068, TXS 0506+056, and the BL Lacs PKS 1424+240 and GB6 J1542+6129.

\subsection{Galactic neutrinos}

Galactic neutrinos could be from the Galactic plane or associated with sources in the Milky Way. A diffuse Galactic neutrino emission is expected from interactions of cosmic rays with interstellar gas and radiation fields that generate pions. The same interactions also give rise to a diffuse highenergy $\gamma$-ray flux from the Galactic plane, which has been measured with Fermi-LAT [31]. While the gamma-ray signal originates from neutral pion decay, the neutrino flux is generated by charged pion decay. The KRA $\mathrm{K}_{\gamma}$ model of the diffuse Galactic flux [32] is in good agreement with gammaray observations. It uses a radially dependent model for the cosmic-ray diffusion coefficient and the advective wind. The primary cosmic-ray spectrum is assumed to have an exponential energy cut-off at $5 \mathrm{PeV}\left(\mathrm{KRA}_{\gamma}^{5}\right)$ or $50 \mathrm{PeV}\left(\mathrm{KRA}_{\gamma}^{50}\right)$ per nucleon. Searches for a diffuse neutrino flux from the Galactic Plane have produced null results thus far, but tight constraints can be placed on diffuse neutrino emission by combining data from IceCube and ANTARES [33].

PWNe are the main gamma-ray emitters in the Galactic plane. Although PWNe gamma-ray emission can be described well with leptonic scenarios, hadronic contributions could be present and give rise to neutrino signals. Using 9.5 years of all-sky IceCube data, a stacking analysis was performed using 35 Galactic PWNe. The analysis resulted in an upper limit on the stacked flux at 90\% C.L. assuming a spectral index of $\gamma=-2.19$ at $\phi_{v_{\mu}+\bar{\nu}_{\mu}}^{90 \% C . L .}=4.57 \times 10^{-12} \mathrm{TeV}^{-1} \mathrm{~cm}^{-2} \mathrm{~s}^{-1}$. Flux bounds on individual candidate sources were also obtained [34].

A separate analysis searched for neutrino emissions from various SNR and gamma-ray sources identified by Milagro and HAWC. No significant excess was observed, and a bound was set using 7 years of IceCube data [35].

\subsection{Solar atmospheric neutrinos}

The Sun is the most extensively studied star over a wide spectrum of wavelengths, yet the energetic Sun $(\mathrm{GeV}-\mathrm{TeV}$ range) remains poorly understood. Cosmic-ray interactions with the solar atmosphere are expected to produce particle showers, which in turn produce neutrinos and gammarays from meson decays. The same underlying production mechanism for solar atmospheric neutrinos and gammarays implies that both are closely connected, yet they have completely different propagation dependencies. The recent detection of gamma-rays from the solar disk with energies above $100 \mathrm{GeV}[36,37]$ has made neutrino searches from the Sun very timely.

Evidence of energetic gamma-ray emissions with $\mathrm{GeV}$ energies from the quiescent Sun was first reported in a 
re-analysis of EGRET data [38]. In 2011, the Fermi-LAT [39] collaboration reported the observation of gamma-ray emissions from the solar disk with energies up to $10 \mathrm{GeV}$. Later studies found that the solar-disk emissions extended to energies beyond $100 \mathrm{GeV}$ and that the flux showed a significant time variation that was anti-correlated with solar activity $[36,37]$. The bright emissions of gamma-rays at energies above $100 \mathrm{GeV}$, which was revealed by analyzing more than 9 years of the Fermi-LAT data, contradict theoretical expectations [37, 40]. Recently, the High Altitude Water Cherenkov (HAWC) gamma-ray observatory searched beyond the sensitivity range of Fermi-LAT (above $200 \mathrm{GeV}$ ). Using 3 years of data, HAWC found no evidence of TeV gamma-ray emissions from the Sun and has set a flux limit [41]. ARGOYBJ reported a limit of above $300 \mathrm{GeV}$ [42]. If the FermiLAT gamma-ray energy spectrum observed during the last solar minimum (2008-2010) can be taken as a representative spectrum that continues into the $\mathrm{TeV}$ range, then the prospects for a first $\mathrm{TeV}$ detection during the current solar minimum (2019-2021) are promising given HAWC's sensitivity.

Given sensitivities of HAWC and ARGO-YBJ, it is not that surprising that no signal was observed, yet. Their current sensitivities are only slightly above the general upper bound on high-energy gamma-ray emission from the Sun [37]. It might require next generation observatories such as the Southern Wide-field Gamma-ray Observatory (SWGO) $[43,44]$ or LHASSO [45] to detect TeV gamma-rays from the Sun.

Recently, the first search for solar atmospheric neutrinos was performed using 7 years of data collected with the IceCube Neutrino Observatory. No evidence for an energetic neutrino flux from the Sun was observed. A $90 \%$ upper limit on the neutrino flux from the Sun was set and is shown in Fig. 5.

The detection of solar atmospheric neutrinos will be important for understanding solar atmospheric magnetic fields, cosmic-ray propagation in the inner solar system, and interactions in the solar atmosphere. For dark matter searches using neutrino signals from self-annihilating dark matter captured by the Sun, characterizing the irreducible background formed by solar atmospheric neutrinos that creates a sensitivity floor to dark matter searches will be important [50-52].

\subsection{Neutrinos and gravitational waves}

Binary black hole (BBH) [53] and binary neutron star (BNS) mergers are confirmed sources of gravitational waves (GW). Relativistic outflows generated in these events could give rise to non-thermal high-energy emissions including highenergy neutrinos. The observation of high-energy neutrinos and gravitational waves from common sources could help to

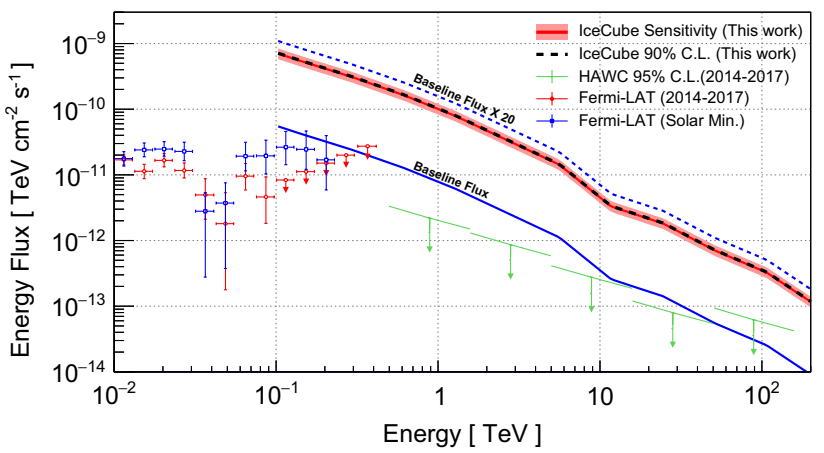

Fig. 5 Preliminary 90\% C.L. neutrino flux upper limit obtained from the solar disk using 7 years of IceCube data [46] compared to the neutrino flux expectation from Ingelman \& Thunman (1996) [47]. The red-shaded band corresponds to the uncertainty of the model. For comparison, the observed gamma-ray flux from Fermi-LAT [37, 48] for the active (solar minimum) and quiescent sun (2014-2017) is shown with the HAWC 95\% C.L. differential flux limit [41, 49]. Figure is taken from Ref. [46]

establish the connection between the dynamics of the progenitor and the properties of the outflow [54].

Searches for coincidences between neutrino telescopes and Advanced LIGO have resulted in no significant candidates during Advanced LIGO from its first observing run Ol (Sep. 12, 2015-Jan. 19, 2016) [54]. A recent search for high-energy neutrino emission coincident with 11 compact binary mergers observed using LIGO-Virgo GW detectors during $O 1$ and $O 2$ found no significant coincidences, and limits on the total isotropic equivalent energy, $E_{i s o}$, emitted as high-energy neutrinos in each GW event were set [55].

The reach of gravitational wave detectors will expand rapidly over the next decade and reach $330 \mathrm{Mpc}$ and $2500 \mathrm{Mpc}$ with O5 for binary neutron star and binary black hole mergers, respectively. The distance range of current and future observing runs is indicated in Fig. 1. Detection with multiple gravitational wave detectors, including KAGRA [56] and VIRGO, reduces the range, but enhances the pointing.

A recent observation of an electromagnetic counterpart [57] for a binary black hole merger detected by LIGO could indicate that the black holes resided inside an AGN disk. The implication of this result is that BBH mergers are also viable multi-messenger targets, further justifying efforts to search for coincidences with neutrinos.

Galactic Core-collapse supernovae (CCSNe) are promising sources of gravitational waves for ground-based detectors. The multi-messenger observation of burst neutrinos, gravitational waves, and photons from the next Galactic CCSN could reveal the supernova burst mechanism, the properties of matter at extreme densities, the interior structures of massive stars, and the origin of many of the chemical elements [58]. A recent review by Murase \& Bartos [59] 
provides more details on these and other multi-messenger observational aspects.

\section{Large-volume water Cherenkov detectors}

Large-volume neutrino detectors with the capability to detect $\sim 10 \mathrm{MeV}$ neutrinos with directional, time, and energy information will take on a unique role as multi-messenger observatories. Not only are they essential for supernova burst detection and location, but also they expand on the successful multi-messenger science program of Super-K [60] and harbors the potential to detect thermal neutrinos from nearby $(<10 \mathrm{Mpc})$ neutron star merger events in coincidence with gravitational waves.

The Hyper-Kamiokande (Hyper-K) experiment [61] is based on the highly successful Super-Kamiokande experiment and takes full advantage of a well-proven water Cherenkov technology. Hyper-K construction started in early 2020, and the experiment is expected to start operations in 2027. The Hyper-K detector tank will be $71 \mathrm{~m}$ in height and $68 \mathrm{~m}$ in diameter with a total volume of $258 \mathrm{kt}$. It is separated into an inner detector region containing $217 \mathrm{kt}$ of water and an outer detector veto region. The inner detector is viewed by an array of 40,000 20-inch PMTs [62]. Hyper$\mathrm{K}$ will be located at the Tochibora site about $8 \mathrm{~km}$ south of the Super-K detector location. The Hyper-K cavern will have a rock overburden of $650 \mathrm{~m}$, which corresponds to a $1750 \mathrm{~m}$ water equivalent (m.w.e.) [62]. Hyper-K will receive a $1.3 \mathrm{MW} v_{\mu}\left(\bar{v}_{\mu}\right)$ beam from J-PARC at an off-axis angle of $2.5^{\circ}$.

The idea to construct a large-volume neutrino detector in Korea has been proposed multiple times. Situated along the J-PARC neutrino beam servicing the Kamioka underground detector sites, a detector in Korea could significantly enhance the science scope of the program [63]. Most recently, a detector in Korea was studied as part of the Hyper-Kamiokande project [64]. There is a very convincing science case for a detector near the $2^{\text {nd }}$ oscillation maximum $(\sim 1100 \mathrm{~km})$, which will result in improved sensitivity to leptonic $\mathrm{CP}$ violation. The higher mass density and longer baseline also result in improved sensitivity to determine the neutrino mass ordering or to test non-standard neutrino interactions [65].

Figure 6 shows potential underground detector locations. The detector location is driven by an optimal positioning for the second oscillation maximum. In addition, a large rock overburden is highly desirable to improve sensitivity to solar neutrinos, supernova neutrinos, and neutrinos of astrophysical origins.

In 2018, a formal organization structure was established, and feasibility studies and detector R\&D started for a largevolume neutrino detector in Korea, known as the Korean

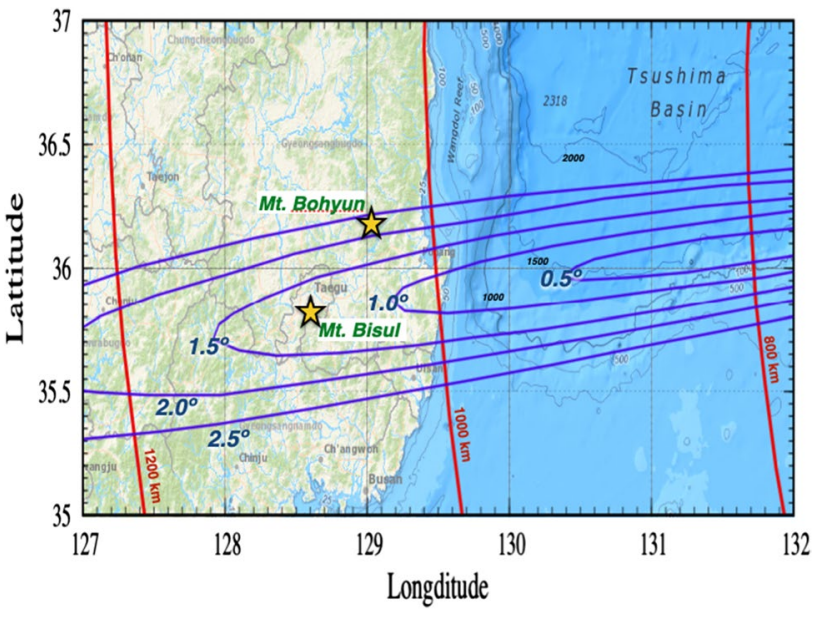

Fig. 6 The two primary candidate sites for $\mathrm{KNO}$ are shown along with the J-PARC off-axis beam angle. Contours are taken from Ref. [63]. The map was obtained from https://maps.ngdc.noaa.gov/ viewers/bathymetry/

Neutrino Observatory (KNO). Funding for feasibility studies of KNO from the Korean government was granted in May 2020, and studies are currently on-going. KNO is now in the preparation stage. If the project is approved, construction could start in 2023 with an expected completion in 2028.

With an off-axis angle (OAA) of $2.5^{\circ}$ at the Hyper-K site, the neutrino interaction rate peaks at an energy of around $0.7 \mathrm{GeV}$. At this energy, the second oscillation maximum occurs at a baseline of roughly $1100 \mathrm{~km}$, with longer baselines corresponding to maxima for higher energies and vice versa [64]. Two primary candidate sites have been identified. The first is at Mt. Bisul (1084 m) in Dal-seong province located near Daegu, is at an OAA of $1.3^{\circ}$ and a $1088 \mathrm{~km}$ baseline, which implies that the neutrino spectrum is more energetic and broader compared to the Hyper-K site. The second candidate site, Mt. Bohyun (1124 m), is located at an OAA of $2.2^{\circ}$, has a baseline of $1040 \mathrm{~km}$, and hosts already the Bohyunsan Optical Astronomy Observatory on its peak. Figure 6 shows the locations of the two primary candidate sites and their location with respect to the beam profile.

The baseline design for KNO has a volume of approximately $250 \mathrm{kt}$ of ultra-pure water and a rock overburden of about $1000 \mathrm{~m}$. Candidate sites were selected to optimize the detection efficiency, and an initial geological exploration was conducted. A construction cost calculation was completed through conceptual design and stability evaluation of the underground facility. Detailed cost estimates are ongoing, and a feasibility study is underway. Designs of critical systems, such as the water purification system are on-going, as is $R \& D$ of photosensors.

Large-volume neutrino detectors have unique sensitivity to core-collapse supernova neutrinos [66-68]. Hyper-K is expected to detect more than 50,000 events for a Galactic 
supernova at $10 \mathrm{kpc}$ and have the capability to detect neutrinos from M31, with about 10-16 burst events expected [62]. Assuming a event reconstruction performance similar to that achieved by Super-K [61], one can expect to reconstruct a Galactic supernova at a $10 \mathrm{kpc}$ distance with about a $1^{\circ}$ to $1.3^{\circ}$ precision. This makes large-volume neutrino detectors essential for distributing early alerts and multi-messenger observations. Even the detection of only a few events from an extragalactic supernova will help reveal the supernova burst mechanism [69].

The diffuse supernova neutrino background (DSNB) [70, 71] left by past supernovae holds important clues about the evolution of the universe, star formation rates, and the supernova burst mechanism. Gadolinium (Gd) doping [72] to tag $\bar{v}_{e}$ between 10 and $30 \mathrm{MeV}$ and thereby distinguish supernova neutrinos from atmospheric neutrino backgrounds can significantly increase the sensitivity to the DSNB.

\section{Conclusions and outlook}

Over the last decade, tremendous progress has been made in neutrino astronomy. The observations of high-energy neutrinos from the direction of the blazar TXS 0506+056 provide strong evidence for cosmic-ray acceleration in AGN jets. However, searches for neutrino emissions from AGN have failed to produce any significant excess, so AGN are not believed to be the dominant source of high-energy neutrinos and cosmic-ray acceleration in the Universe. Further observations with neutrinos will be necessary to identify regions of cosmic-ray acceleration in jets and understand underlying acceleration mechanisms.

At energies above $10 \mathrm{PeV}$, the detection of neutrinos via radio is extremely promising. The IceCube-Gen2 facility will contain a surface radio array to extend the energy range accessible via the in-ice optical sensor array by several orders of magnitude and allow for cross-calibration between radio and optical neutrino detection [8]. Radio emission is generated by particle showers through the Askaryan effect [73] when neutrinos interact in the ice. The technique has been proposed for ARIANNA [74] and is the basis for the high-energy neutrino searches with ANITA [75]. An alternative method is proposed for the Giant Radio Array for Neutrino Detection (GRAND) [76] to detect ultra-highenergy neutrinos via the extensive air showers induced by the decay of tau leptons created in the interaction of tau neutrinos under Earth's surface.

Worldwide efforts are underway to construct the next generation of neutrino telescopes and large-volume neutrino detectors. These detectors are expected to identify the dominant source populations and quantify their respective contributions to the diffuse astrophysical neutrino flux. The outlook for neutrino multi-messenger observations is bright and further improves with reduced neutrino pointing uncertainty, larger neutrino detector volumes and effective areas, and improved reach of gamma-ray, optical, and gravitational wave detectors.

Acknowledgements C. Rott acknowledges support from the National Research Foundation of Korea (NRF) for the Basic Science Research Program NRF-2020R1A2C3008356.

Open Access This article is licensed under a Creative Commons Attribution 4.0 International License, which permits use, sharing, adaptation, distribution and reproduction in any medium or format, as long as you give appropriate credit to the original author(s) and the source, provide a link to the Creative Commons licence, and indicate if changes were made. The images or other third party material in this article are included in the article's Creative Commons licence, unless indicated otherwise in a credit line to the material. If material is not included in the article's Creative Commons licence and your intended use is not permitted by statutory regulation or exceeds the permitted use, you will need to obtain permission directly from the copyright holder. To view a copy of this licence, visit http://creativecommons.org/licenses/by/4.0/.

\section{References}

1. A. De Angelis, G. Galanti, M. Roncadelli, Mon. Not. Roy. Astron. Soc. 432, 3245 (2013). (1302.6460)

2. M.G. Aartsen et al., (IceCube Collaboration), Phys. Rev. Lett. 113, 101101 (2014)

3. M. G. Aartsen et al., (IceCube Collaboration), Science 342, 1242856 (2013). arXIV: 1311.5238

4. M. Aartsen et al., (IceCube, Fermi-LAT, MAGIC, AGILE, ASASSN, HAWC, H.E.S.S., INTEGRAL, Kanata, Kiso, Kapteyn, Liverpool Telescope, Subaru, Swift NuSTAR, VERITAS, VLA/17B-403 Collaborations), Science 361, eaat1378 (2018a). arXIV: 1807.08816

5. M. Aartsen et al., (IceCube Collaboration), Science 361, 147 (2018b). arXIV: 1807.08794

6. S. Adrian-Martinez et al., (KM3NeT Collaboration), J. Phys. G 43, 084001 (2016). arXIV: 1601.07459

7. A. Avrorin et al., (Baikal-GVD Collaboration), EPJ Web Conf. 191, 01006 (2018). arXIV: 1808.10353

8. M. Aartsen et al., (IceCube-Gen2 Collaboration) (2020a). arXIV : 2008.04323

9. M. Agostini et al., (P-ONE Collaboration), Nat. Astron. 4, 913 (2020). arXIV: 2005.09493

10. W. Kang, C. Tönnis, C. Rott, (IceCube Collaboration), PoS ICRC2019, 928 (2020). arXIV: 1908.07734

11. M. Aartsen et al., (IceCube Collaboration), J. Phys. G 44, 054006 (2017a). arXIV: 1607.02671

12. L. Classen, C. Dorn, A. Kappes, T. Karg, M. Kossatz, A. Kretzschmann, H.-W. Ortjohann, J. Reubelt, K.-H. Sulanke, R. Weigel, PoS ICRC2019, 855 (2019)

13. K. Murase, E. Waxman, Phys. Rev. D 94, 103006 (2016). arXIV: 1607.01601

14. A. Silvestri, S.W. Barwick, Phys. Rev. D 81, 023001 (2010). arXIV: 0908.4266

15. M. Aartsen et al., (IceCube Collaboration), Eur. Phys. J. C 79, 234 (2019a). arXIV: 1811.07979

16. K. Murase, M. Fukugita, Phys. Rev. D 99, 063012 (2019). arXIV : 1806.04194

17. M. Ageron et al., (ANTARES Collaboration), Nucl. Instrum. Meth. A 656, 11 (2011). arXIV: 1104.1607 
18. D. Samtleben, Neutrinos in the mediterranean sea (2020), https ://doi.org/10.5281/zenodo.3959672

19. R. Abbasi et al., (IceCube Collaboration), (2020). arXIV: 2011.03545

20. M. Santander, (IceCube Collaboration), Neutrino2020, (2020). https://doi.org/10.5281/zenodo.3959674

21. M. Aartsen et al., (IceCube Collaboration), Phys. Rev. Lett. 125, 121104 (2020b), arXIV: 2001.09520

22. J. Stettner, (IceCube Collaboration), PoS ICRC2019, 1017 (2020). arXIV: 1908.09551

23. M. Aartsen et al., (IceCube Collaboration), Phys. Rev. D 99, 032004 (2019b). arXIV: 1808.07629

24. A. Schneider, PoS ICRC2019, 1004 (2020). http://arxiv.org/ abs/1907.11266

25. S. Paiano, R. Falomo, A. Treves, R. Scarpa, Astrophys. J. Lett. 854, L32 (2018). arXIV: 1802.01939

26. R. Stein et al., (2020). arXIV: 2005.05340

27. N. Senno, K. Murase, P. Meszaros, Astrophys. J. 838, 3 (2017). arXIV: 1612.00918

28. L. Dai, K. Fang, Mon. Not. Roy. Astron. Soc. 469, 1354 (2017). arXIV: 1612.00918

29. C. Lunardini, W. Winter, Phys. Rev. D 95, 123001 (2017). arXIV : 1612.03160

30. M. Aartsen et al., (IceCube Collaboration), Phys. Rev. Lett. 124, 051103 (2020c). arXIV: 1910.08488

31. M. Ackermann et al., (Fermi-LAT Collaboration), Astrophys. J. 750, 3 (2012). arXIV: 1202.4039

32. D. Gaggero, D. Grasso, A. Marinelli, A. Urbano, M. Valli, Astrophys. J. Lett. 815, L25 (2015). arXIV: 1504.00227

33. A. Albert et al., (ANTARES, IceCube Collaborations), Astrophys. J. Lett. 868, L20 (2018a). arXIV: 1808.03531

34. M. Aartsen et al., Astrophys. J. 898, 117 (2020d). arXIV: 2003.12071

35. M. Aartsen et al., (IceCube Collaboration), Astrophys. J. 849, 67 (2017b). arXIV: 1707.03416

36. K.C.Y. Ng, J.F. Beacom, A.H.G. Peter, C. Rott, Phys. Rev. D 94, 023004 (2016b). arXIV: 1508.06276

37. T. Linden, B. Zhou, J.F. Beacom, A.H. Peter, K.C. Ng, Q.-W. Tang, Phys. Rev. Lett. 121, 131103 (2018). arXIV:1803.05436

38. E. Orlando, A.W. Strong, Astron. Astrophys. 480, 847 (2008). arXIV: 1508.06276

39. A. Abdo et al., (Fermi-LAT Collaboration), Astrophys. J. 734, 116 (2011). arXIV:1104.2093

40. Q.-W. Tang, K.C. Ng, T. Linden, B. Zhou, J.F. Beacom, A.H. Peter, Phys. Rev. D 98, 063019 (2018a). arXIV:1804.06846

41. A. Albert et al., (HAWC Collaboration), Phys. Rev. D98, 123011 (2018b). arXIV:1808.05620

42. B. Bartoli et al., (ARGO-YBJ Collaboration), Astrophys. J. 872, 143 (2019) arXIV:1901.04201

43. P. Abreu et al. (2019). arXIV: 1907.07737

44. A. Albert et al. (2019a). arXIV: 1902.08429

45. X. Bai et al., (2019). arXIV: 1905.02773

46. M. Aartsen et al., (IceCube Collaboration), JCAP 2102, 025 (2021). arXIV: 1912.13135

47. G. Ingelman, M. Thunman, Phys. Rev. D 54, 4385 (1996). (hep-ph/9604288)

48. Q.-W. Tang, K.C.Y. Ng, T. Linden, B. Zhou, J.F. Beacom, A.H.G. Peter, Phys. Rev. D 98, 063019 (2018b). arXIV: 1804.06846

49. B. Zhou, K.C.Y. Ng, J.F. Beacom, A.H.G. Peter, Phys. Rev. D 96, 023015 (2017). arXIV: 1612.02420
50. K.C.Y. Ng, J.F. Beacom, A.H.G. Peter, C. Rott, Phys. Rev. D 96, 103006 (2017). arXIV: 1703.10280

51. C.A. Argüelles, G. de Wasseige, A. Fedynitch, B.J.P. Jones, JCAP 1707, 024 (2017). arXIV: 1703.07798

52. J. Edsjö, J. Elevant, R. Enberg, C. Niblaeus, JCAP 1706, 033 (2017). arXIV: 1704.02892

53. B. Abbott et al., (LIGO Scientific, Virgo Collaborations), Phys. Rev. Lett. 116, 061102 (2016). arXIV: 1602.03837

54. A. Albert et al., (ANTARES, IceCube, LIGO, Virgo Collaborations), Astrophys. J. 870, 134 (2019b). arXIV: 1810.10693

55. M. Aartsen et al., (IceCube Collaboration), Astrophys. J. Lett. 898 , L10 (2020e). arXIV: 2004.02910

56. T. Akutsu et al., (KAGRA Collaboration), Nat. Astron. 3, 35 (2019). arXIV: 1811.08079

57. M. Graham et al., Phys. Rev. Lett. 124, 251102 (2020). arXIV: 2006.14122

58. D. Radice, V. Morozova, A. Burrows, D. Vartanyan, H. Nagakura, Astrophys. J. 876, L9 (2019). https://doi.org/10.3847\%2F204 $1-8213 \% 2 \mathrm{Fab} 191 \mathrm{a}$

59. K. Murase, I. Bartos, Ann. Rev. Nucl. Part. Sci. 69, 477 (2019). arXIV: 1907.12506

60. K. Abe et al., (Super-Kamiokande Collaboration), Astrophys. J. Lett. 830, L11 (2016). arXIV: 1608.08745

61. K. Abe et al., (Hyper-Kamiokande Collaboration) (2018a). arXIV : 1805.04163

62. K. Abe et al., (Hyper-Kamiokande Collaboration) (2020). arXIV : 2009.00794

63. K. Hagiwara, N. Okamura, K.-i. Senda, Phys. Lett. B 637, 266 (2006). note [Erratum: Phys.Lett.B 641, 491 (2006)]. hep-ph/0504061

64. K. Abe et al., (Hyper-Kamiokande Collaboration), PTEP 2018 , 063C01 (2018b). arXIV: 1611.061184

65. J. Liao, D. Marfatia, K. Whisnant, JHEP 01, 071 (2017). arXIV: 1612.01443

66. K. Hirata et al., (Kamiokande-II Collaboration), Phys. Rev. Lett. $\mathbf{5 8}, 1490(1987)$

67. M. Ikeda et al., (Super-Kamiokande Collaboration), Astrophys. J. 669, 519 (2007). arXIV: 0706.2283

68. K. Nakamura, S. Horiuchi, M. Tanaka, K. Hayama, T. Takiwaki, K. Kotake, Mon. Not. Roy. Astron. Soc. 461, 3296 (2016). arXIV : 1602.03028

69. T.A. Thompson, J.L. Prieto, K. Stanek, M.D. Kistler, J.F. Beacom, C.S. Kochanek, Astrophys. J. 705, 1364 (2009). (0809.0510)

70. J.F. Beacom, Ann. Rev. Nucl. Part. Sci. 60, 439 (2010). arXIV: 1004.3311

71. C. Lunardini, Astropart. Phys. 79, 49 (2016). arXIV: 1007.3252

72. J.F. Beacom, M.R. Vagins, Phys. Rev. Lett. 93, 171101 (2004). (hep-ph/0309300)

73. G. Askar'yan, Sov. Phys. JETP 14, 441 (1962)

74. A. Anker et al. (2020). arXIV: 2004.09841

75. P. Gorham et al., (ANITA Collaboration), Phys. Rev. Lett. 99, 171101 (2007). (hep-ex/0611008)

76. J. Álvarez Muñiz et al., (GRAND Collaboration), Sci. China Phys. Mech. Astron. 63, 219501 (2020). arXIV: 1810.09994

Publisher's Note Springer Nature remains neutral with regard to jurisdictional claims in published maps and institutional affiliations. 